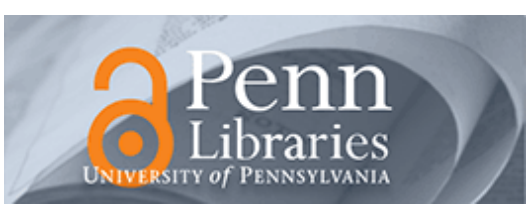

University of Pennsylvania
ScholarlyCommons

Research Briefs

Leonard Davis Institute of Health Economics

4-3-2019

\title{
The Effect of Predictive Analytics-Driven Interventions on Healthcare Utilization
}

Guy David

University of Pennslyvania

Aaron Smith-McLallen

Independence Blue Cross of Philadelphia

Benjamin Ukert

University of Pennsylvania

Follow this and additional works at: https://repository.upenn.edu/ldi_researchbriefs

David, Guy; Smith-McLallen, Aaron; and Ukert, Benjamin. The Effect of Predictive Analytics-Driven Interventions on Healthcare Utilization. LDI Research Briefs. 2019; No. 54. https://ldi.upenn.edu/brief/effect-predictive-analytics-driven-interventionshealthcare-utilization

$">$

https://ldi.upenn.edu/brief/effect-predictive-analytics-driven-interventions-healthcare-utilization 


\title{
The Effect of Predictive Analytics-Driven Interventions on Healthcare Utilization
}

\begin{abstract}
Among high-risk Medicare Advantage members with congestive heart failure, a proactive outreach program driven by a claims-based predictive algorithm reduced the likelihood of an emergency department (ED) or specialist visit in one year by $20 \%$ and $21 \%$, respectively. The average number of visits dropped as well, with a $40 \%$ reduction in the volume of ED visits and a $27 \%$ reduction in the volume of cardiology visits after the first year.
\end{abstract}

\section{Keywords}

predictive analytics, health care utilization, risk score

\section{License}

Creative

This worksis licensed under a Creative Commons Attribution-No Derivative Works 4.0 License. License 
DATA DRIVEN.POLICY FOCUSED

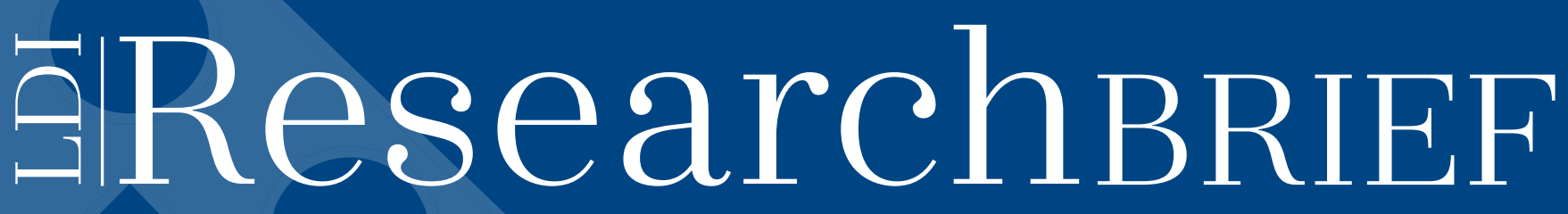

Research to Improve the Nation's Health System

2019. No. 3

\section{THE EFFECT OF PREDICTIVE ANALYTICS-DRIVEN INTERVENTIONS ON HEALTHCARE UTILIZATION}

\section{Significant reductions in emergency department and specialist visits}

Guy David, Aaron Smith-McLallen, Benjamin Ukert

Journal of Health Economics - March 2019

\section{KEYFINDINGS}

Among high-risk Medicare Advantage members with congestive heart failure, a proactive outreach program driven by a claims-based predictive algorithm reduced the likelihood of an emergency department (ED) or specialist visit in one year by $20 \%$ and $21 \%$, respectively. The average number of visits dropped as well, with a $40 \%$ reduction in the volume of ED visits and a $27 \%$ reduction in the volume of cardiology visits after the first year.

\section{THE QUESTION}

The typical Medicare beneficiary receives care from eight providers per year, while the top ten percent have seen over 21 providers per year. For chronically ill individuals, care fragmentation can be especially damaging and can lead to adverse health outcomes. One proactive approach is the use of predictive analytics to identify and target high-risk beneficiaries in real time. Paired with an effective care management strategy, analytics can be used to close gaps in needed care, and reduce unnecessary hospitalizations and emergency visits.

In this study, the authors evaluate a care coordination intervention administered by Independence Blue Cross of Philadelphia to their Medicare Advantage members with congestive heart failure. Members were assigned a risk score by a predictive algorithm that draws on medical and pharmacy claims, lab results, demographic information, and more. Those with the highest risk scores were selected for telephone outreach by a care management team; the total number selected was determined by available resources,

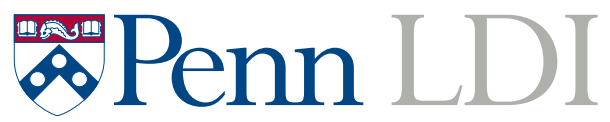

LEONARD DAVIS INSTITUTE of HEALTH ECONOMICS rather than illness severity. This allowed the researchers to follow a comparison group of members with similar risk scores who did not receive the intervention.

The two questions addressed in the study are: (1) whether the risk score could predict hospitalizations and ED visits, and (2) how the care management intervention affected inpatient and outpatient care use 30, 90,180 , and 360 days after outreach.

\section{THE RESULTS}

The final sample included 1,974 patients, half of whom were targeted for the intervention. The authors found that the risk score is a strong predictor of hospitalization and ED visits at all time windows. As shown in the Figure, among those not selected for outreach, the number of hospitalizations and ED visits decrease as relative distance from the cutoff increases.

The targeted group saw large reductions in the likelihood of a cardiologist and ED visit. After 30 days, the probability of a cardiologist visit decreased by 14 percentage points, and this effect persists over the first year. This represents a $20 \%$ reduction in cardiologist visits relative to the 360-day control group average. The probability of an ED visit decreased 


\section{ResearchBRIEF}

Figure Fraction of Hospitalizations and ED Visits for Members Not Selected for Outreach at 360 days and Risk Score Rank (with 1 being highest risk score and 5000 being the lowest risk score).

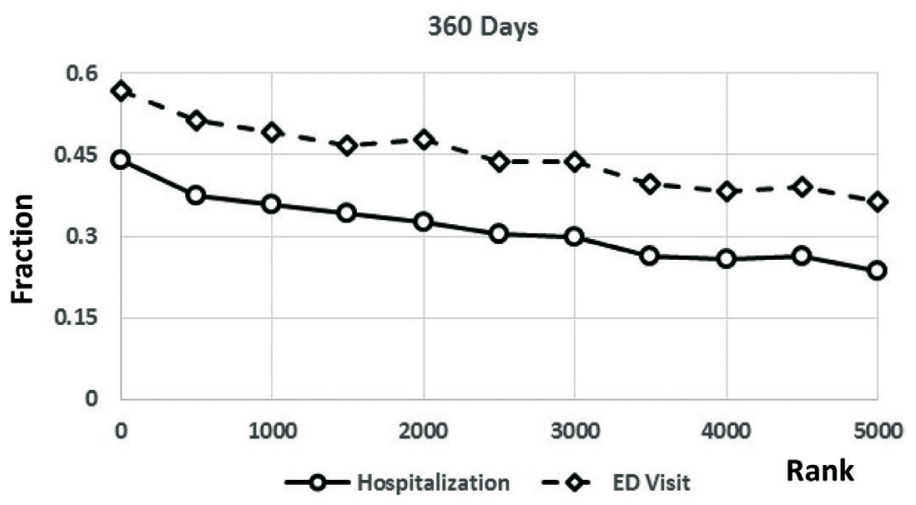

by about 16 percentage points (38\%) and 12 percentage points (21\%) after 180 and 360 days, respectively. The intervention had no impact on primary care physician visits, regardless of time period. Though statistically insignificant, the program reduced the probability of hospital admission by 8.4 percentage points after 90 days, and 3.8 percentage points after 360 days. This result is economically meaningful given that even small reductions in admission rates can lead to significant cost savings.

The authors also studied the overall volume of hospitalizations, primary care visits, cardiologist visits, ED visits, length of stay, and hospitalization costs over time, and found significant reductions only in cardiologist visits and ED visits. After 360 days, the rate of ED visits decreased by 0.52 visits (a $40 \%$ reduction in the average) and the number of cardiologist visits decreased by 0.70 visits (a $27 \%$ reduction in the average). Though statistically insignificant, hospital admissions decreased by $38 \%$ at 30 days, $46 \%$ at 90 days, $26 \%$ at 180 days, and $20 \%$ after 360 days relative to the control group average, which could translate to potential cost savings.

\section{THE IMPLICATIONS}

These findings suggest that continuity of care, in the form of a communication hub as part of a targeted intervention, may serve as an effective tool in reducing unnecessary utilization among high-risk populations. Additionally, effective telehealth case management, rather than face to face case management, can lead to reductions in ED care.
Since the number and likelihood of primary care visits was not affected by outreach, the findings may have been driven by improved care coordination among fewer providers. Intensity of treatment and patient involvement may also have changed as a result of outreach and may explain the findings.

The study also demonstrate how predictive analytics can be used to identify high-risk patients for effective interventions. A predictive algorithm can draw information from hospital discharge data and electronic health records (primarily used by providers) and information on medication adherence and health care utilization (primarily used by payers). A coordinated approach to analytics may help payers and providers realize efficiencies in care and direct potentially timely interventions to patients in greatest need.

The results imply savings 30 days post-outreach of $\$ 500$ to $\$ 1,000$ for ED visits per member per year. Similarly, while mostly statistically insignificant, the reduction in hospitalization implies annual savings of about $\$ 13,000$ per member over the 30 days after outreach. These generally large effects may be the result of targeting the sickest members within a high spending population, who based on these predictive models have the most to gain from an intervention.

\section{THE STUDY}

The authors analyzed claims data for 29,222 fully-insured Medicare Advantage members diagnosed with congestive heart failure. The algorithm was run in 10 waves between May 2012 and May 2015, and members did not appear in more than one wave. To measure the effect of the outreach program on health care utilization, the authors compared individuals selected for outreach and formed a similar sized control group consisting of individuals whose risk score rankings fell just below the treatment cut off. They conduct several robustness checks to test the validity of the study design and sample selection.

Outreach was initiated through phone calls by registered nurse health coaches, but not all outreach resulted in enrollment in a care management program. The program included a needs assessment by the health coach, and assistance coordinating care, scheduling appointments, accessing medications, arranging transportation, and connecting with social workers and behavioral health specialists when needed.

David G, Smith-McLallen A, Ukert B. The Effect of Predictive AnalyticsDriven Interventions on Healthcare Utilization. Journal of Health Economics. DOl: https://doi.org/10.1016/j.jhealeco.2019.02.002

\section{LEAD AUTHOR}

\section{DR. GUY DAVID}

Guy David, PhD is the Gilbert and Shelley Harrison Associate Professor of Health Care Management at Penn's Wharton School. He pays attention to aspects of the health care system that are understudied, just emerging, or difficult to measure. His research on emergency medical services, home health care, primary care, specialty hospitals, and ambulatory surgery centers has yielded insights into how individuals, firms, and regulators interact across the continuum of care.

His study co-authors are Aaron Smith-McLallen, PhD, Director of Data Science and Health Care Analytics at Independence Blue Cross, and Benjamin Ukert, PhD, Senior Health Economist at HealthCore Inc. 